Brief Report

\title{
Prevalence of SARS-CoV-2 Variants of Concern and Variants of Interest in COVID-19 Breakthrough Infections in a Hospital in Monterrey, Mexico
}

\author{
Kame A. Galán-Huerta ${ }^{1,+} \mathbb{D}$, Samantha Flores-Treviño ${ }^{2,+} \mathbb{D}$, Daniel Salas-Treviño ${ }^{2} \mathbb{D}$, Paola Bocanegra-Ibarias ${ }^{2} \mathbb{D}$, \\ Ana M. Rivas-Estilla ${ }^{1}{ }^{1}$, Eduardo Pérez-Alba ${ }^{2} \mathbb{D}$, Sonia A. Lozano-Sepúlveda ${ }^{1}$, Daniel Arellanos-Soto ${ }^{1}$ and \\ Adrián Camacho-Ortiz ${ }^{2, *}$ \\ check for \\ updates \\ 1 Center for Investigation and Innovation in Medical Virology, Department of Biochemistry and Molecular \\ Medicine, School of Medicine, Universidad Autónoma de Nuevo León, Monterrey 64460, Mexico; \\ kame.galanhr@uanl.edu.mx (K.A.G.-H.); amrivas1@yahoo.ca (A.M.R.-E.); \\ lozano_sonia@hotmail.com (S.A.L.-S.); d_arellanos_s@yahoo.com (D.A.-S.) \\ 2 Department of Infectious Diseases, School of Medicine, University Hospital “Dr. José Eleuterio González", \\ Universidad Autónoma de Nuevo León, Monterrey 64460, Mexico; samflorest@gmail.com (S.F.-T.); \\ danielsalast91@gmail.com (D.S.-T.); paola.bocanegraib@gmail.com (P.B.-I.); \\ md.eduardo.perez@gmail.com (E.P.-A.) \\ * Correspondence: acamacho_md@yahoo.com \\ + These authors contributed equally to this work.
}

Citation: Galán-Huerta, K.A.;

Flores-Treviño, S.; Salas-Treviño, D. Bocanegra-Ibarias, P.; Rivas-Estilla, A.M.; Pérez-Alba, E.;

Lozano-Sepúlveda, S.A.;

Arellanos-Soto, D.; Camacho-Ortiz, A.

Prevalence of SARS-CoV-2 Variants

of Concern and Variants of Interest in COVID-19 Breakthrough Infections in a Hospital in Monterrey, Mexico. Viruses 2022, 14, 154. https:// doi.org/10.3390/v14010154

Academic Editors: Eurico Arruda, Helena Lage Ferreira, José Luiz Proença Módena and William Marciel de Souza

Received: 10 November 2021

Accepted: 11 January 2022

Published: 14 January 2022

Publisher's Note: MDPI stays neutral with regard to jurisdictional claims in published maps and institutional affiliations.

Copyright: (C) 2022 by the authors. Licensee MDPI, Basel, Switzerland. This article is an open access article distributed under the terms and conditions of the Creative Commons Attribution (CC BY) license (https:// creativecommons.org/licenses/by/ $4.0 /)$

\begin{abstract}
SARS-CoV-2 variants of concern (VOCs) or of interest (VOIs) causing vaccine breakthrough infections pose an increased risk to worldwide public health. An observational case-control study was performed of SARS-CoV-2 vaccine breakthrough infections in hospitalized or ambulatory patients in Monterrey, Mexico, from April through August 2021. Vaccination breakthrough was defined as a SARS-CoV-2 infection that occurred any time after 7 days of inoculation with partial (e.g., first dose of two-dose vaccines) or complete immunization (e.g., second dose of two-dose vaccines or single-dose vaccine, accordingly). Case group patients $(n=53)$ had partial or complete vaccination schemes with CanSino (45\%), Sinovac (19\%), Pfizer/BioNTech (15\%), and AstraZeneca/Oxford (15\%). CanSino was administered most frequently in ambulatory patients $(p<0.01)$. The control group $(n=19)$ received no COVID-19 vaccines. Among SARS-CoV-2 variants detected by whole-genome sequencing, VOC Delta B.1.617.2 predominated in vaccinated ambulatory patients $(p<0.01)$ and AY.4 in hospitalized patients ( $p=0.04)$; VOI Mu B.1.621 was detected in four $(7.55 \%)$ vaccinated patients. SARS-CoV-2 breakthrough infections in our hospital occurred mostly in patients vaccinated with CanSino due to the higher prevalence of CanSino vaccine administration in our population. These patients developed mild COVID-19 symptoms not requiring hospitalization. The significance of this study lies on the detection of SARS-CoV-2 variants compromising the efficacy of local immunization therapies in Monterrey, Mexico.
\end{abstract}

Keywords: SARS-CoV-2; COVID-19; Latin America; Mexico; COVID-19 vaccines; surveillance; variant of concern; Delta; Mu; CanSino

\section{Introduction}

The coronavirus disease 2019 (COVID-19) emerged in December 2019 and was later declared by the World Health Organization as a pandemic [1]. COVID-19 is caused by the severe acute respiratory syndrome coronavirus 2 (SARS-CoV-2), and there are currently more than 200 million cases and over 4 million deaths reported worldwide. In Mexico, 3 million cases and up to 250,000 deaths have been reported [2].

The rapid development of efficient and safe vaccines against COVID-19 undertook countless efforts in the year 2020 [3]. Nowadays, up to 4.5 billion vaccine doses have been already administered worldwide [2]. Mexico started administering the BNT162b2 
(Pfizer/BioNTech) vaccine to its health personnel in late December of 2020, soon after it was declared for emergency use by the WHO. BNT162b2 is a lipid nanoparticle-encapsulated nucleoside-modified RNA vaccine that encodes the full-length surface spike (S) glycoprotein of SARS-CoV-2 [4,5]. Since then, Ad5-nCoV (CanSino Biologics Inc.) and AZD1222 (AstraZeneca/University of Oxford), both nonreplicating recombinant adenovirus vectors that express the $S$ protein [4-7], and the (Vero cell) inactivated whole-virus CoronaVac (Sinovac Life Sciences) vaccine [8] have also been administered in the country. Nevertheless, governmental vaccine acquisition has been laborious, and reduced widespread vaccine availability and delayed vaccine uptake have caused only 85.3 million vaccine doses to be administered in Mexico [9].

Moreover, SARS-CoV-2 variants have emerged in several places of the world carrying several mutations in the $S$ protein [10]. These variants are classified by the WHO as variants of concern (VOCs) and variants of interests (VOIs). Variants of Concern can either have increased transmissibility, virulence, severe disease presentation, reduced diagnostic detection, vaccine effectiveness, or treatment effectiveness. Current VOCs are Alpha (B.1.1.7), Beta (B.1.351), Gamma (P.1), Delta (B.1.617.2), and Omicron (B.1.1.529). The VOIs have genetic changes that affect transmissibility, disease presentation, and immune and therapeutic effectiveness, in addition to significant community transmission posing a risk to global public health. The current VOIs designated by WHO are Lambda (C.37) and $\mathrm{Mu}$ (B.1.621) [11]. Such variants can confer superior viral replication or transmission and compromise the efficacy of current immunization therapies [7]. Thus, the transmission of these variants is worrisome as they pose an increased risk to worldwide public health $[7,10]$.

The aim of this study was to describe the prevalence of SARS-CoV-2 VOCs or VOIs in patients with partial or complete vaccination schemes in Monterrey, Mexico.

\section{Materials and Methods}

\subsection{Setting}

The University Hospital “Dr. José Eleuterio Gonzalez" located in Monterrey, Mexico, is a multi-building hospital and medical school complex that comprises two separate hospitals. The main building is a 600-bed hospital designated for the treatment of non-COVID-19 cases. It has an average of 25,000 admissions per year. The second building is an 85-bed hospital that was designated and first used for the diagnosis and treatment of suspected or confirmed COVID-19 cases. It has an outpatient clinic, an emergency room, hospitalization wards, an intensive care unit (ICU), a step-down unit, and two operating rooms.

\subsection{Selection Criteria}

The design is an observational case-control study of SARS-CoV-2 vaccine breakthrough infections in hospitalized patients in our unit or who attended the clinic from April through August 2021. Individuals with COVID-19 confirmed by detection of SARS-CoV-2 by realtime reverse transcription PCR (rRT-PCR) were included. The case group was composed of individuals aged $>18$ years with a positive rRT-PCR for SARS-CoV-2 and $C t \leq 30$, either hospitalized (i.e., with supplemental oxygen requirement and abnormal chest imaging) or ambulatory patients (i.e., with mild symptoms and without oxygen requirement). The infection occurred any time after 7 days of inoculation with the first dose of Pfizer/BioNTech, AstraZeneca/Oxford, or CoronaVac, or with the single dose CanSino, according to patients records. The control group consisted of randomly selected SARS-CoV-2-positive cases that were also hospitalized occurred in the same period as the breakthrough infections.

\subsection{Whole-Genome Sequencing and Lineage Determination}

RNA was extracted from $200 \mu \mathrm{L}$ of nasopharyngeal swab specimens transported in UTM viral transport medium (COPAN Diagnostics Inc., Murrieta, CA, USA) using a STARMag Universal Cartridge Kit (Seegene Technologies, Seoul, South Korea) on a Nimbus extraction workstation (Seegene Technologies) following the manufacturer's instructions. SARS-CoV-2 detection was performed with a Smart Detect SARS-CoV-2 rRT-PCR Kit (InBios 
International, Inc., Seattle, WA, USA) on a CFX96 thermal cycler (Bio-Rad Laboratories, Hercules, CA, USA).

The improved ARTIC multiplex PCR method for SARS-CoV-2 genome sequencing was performed using nanopore technology [12]. Viral RNA $(8 \mu \mathrm{L})$ was converted to cDNA with LunaScript RT (New England Biolabs, Ipswich, MA, USA) and later used in a multiplex PCR using Q5 Hot Start HF polymerase (New England Biolabs). Ends were repaired with NEBNext Ultra II end repair/dA-tailing module (New England Biolabs), and native barcodes (Oxford Nanopore Technologies, Oxford, United Kingdom) were added with Blunt/TA ligase (New England Biolabs). After washing with AMPure XP beads (Beckman Coulter, Brena, CA, USA), adapters (Oxford Nanopore Technologies, Oxford, United Kingdom) were added with the NEBNext quick ligation module (New England Biolabs). The library was sequenced on an R9.4.1 flow cell (Oxford Nanopore Technologies, Oxford, United Kingdom) for $12 \mathrm{~h}$. The sequences were assembled using the ARTIC network bioinformatics pipeline via nanopolish and the reference sequence MN908947.3 to obtain the consensus sequences. SARS-CoV-2 lineages were obtained using the Pangolin COVID-19 Lineage Assigner web application (https:/ / pangolin.cog-uk.io/, accessed on 6 September 2021) [13]. Sequences with more than $10 \%$ of Ns were discarded from the study.

\subsection{Phylogenetic Analyses}

Global reference sequences were selected from the global dataset $(n=3493)$ obtained from Nextstrain [14] and were retrieved from the Global Initiative for Sharing All Influenza Data (GISAID) database. Sequence quality was determined with Nextclade web tool, and 58 low-quality sequences were removed. The final sequence dataset was composed of 3499 sequences ( 3435 from the global dataset and 64 generated from this study). Afterward, Nextstrain was used to analyze these sequences. Phylogenetic trees were visualized with ggtree [15]. The authors of sequences used are listed in Supplementary Table S1. The sequences generated were deposited in GISAID (EPI_ISL_4889733-EPI_ISL_4889791 and EPI_ISL_4926951-EPI_ISL_4926955).

\subsection{Statistical Analyses}

Continuous variables, such as age and Ct values, are expressed as means. The dispersion of these variables is expressed in interquartile values. The statistical mean differences were evaluated using the one-way ANOVA test. The normal distribution of these variables was verified using the Kolmogorov-Smirnoff test. Contingency tables using the $\chi^{2}$ and Fisher's exact statistical tests $(2 \times 2$ tables $)$ were used to evaluate discontinuous variables. In all tests, a $p$-value $<0.05$ was considered significant.

\section{Results}

\subsection{Breakthrough Infections in Monterrey, Mexico}

A total of 53 cases of COVID-19 vaccine breakthrough infections occurred in our hospital from 19 April 2021 to 11 August 2021 (Supplementary Table S2). The definition for vaccination breakthrough in this study was any SARS-CoV-2 infection that occurred any time after 7 days of inoculation with partial (e.g., first dose of two-dose vaccines) or complete immunization (e.g., second dose of two-dose vaccines or one single-dose vaccine, accordingly). These patients were vaccinated with CanSino $(n=24,45.28 \%)$ between March and May, Sinovac $(n=10,18.87 \%)$ between April and June, and equally with Pfizer/BioNTech $(n=8,15.09 \%)$ between January and August and AstraZeneca/Oxford $(n=8,15.09 \%)$ between April and May. The comparison of vaccinated ambulatory patients $(n=30,56.60 \%)$ and those who required hospitalization $(n=23,43.40 \%)$ showed that the single-dose CanSino was administered most frequently in ambulatory patients $(p<0.01)$. Furthermore, almost all ambulatory patients received complete vaccination schemes regardless of vaccine type before they developed COVID-19-related symptoms, unlike hospitalized patients $(p=0.03)$. 
In the study, 19 unvaccinated hospitalized patients (control group) were also included in the same period as the case-control group. Hospitalized vaccinated patients had a higher median age $(p<0.01)$ and comorbidities frequency, such as hypertension $(n=11,47.82 \%$, $p=0.02)$ and diabetes mellitus type $2(n=13,56.52 \%, p<0.01)$, compared to ambulatory or unvaccinated hospitalized patients. No differences were found between vaccinated (hospitalized and ambulatory) and unvaccinated patients regarding oxygen requirement, clinical intervention, or hospital treatment (Table 1).

Table 1. Baseline data, comorbidities, vaccination status, and clinical intervention of patients included.

\begin{tabular}{|c|c|c|c|c|}
\hline \multirow{2}{*}{ Characteristic $^{1}$} & \multicolumn{2}{|c|}{$\begin{array}{c}\text { Vaccinated } \\
n \text { (\% or Range) }\end{array}$} & \multirow{2}{*}{$\begin{array}{c}\text { Unvaccinated } \\
n(\% \text { or Range) } \\
\text { Hospitalized } \\
(n=19)\end{array}$} & \multirow{2}{*}{$p$-Value ${ }^{2}$} \\
\hline & $\begin{array}{l}\text { Hospitalized } \\
\quad(n=23)\end{array}$ & $\begin{array}{l}\text { Ambulatory } \\
\quad(n=30)\end{array}$ & & \\
\hline Male & $12(52.17)$ & $13(43.33)$ & $9(47.37)$ & \\
\hline Female & $11(47.83)$ & $17(56.67)$ & $10(52.63)$ & \\
\hline Median age (IQR), years & $59.70(50-70)$ & $\begin{array}{c}42.80 \\
(27.75-56.25)\end{array}$ & $48.47(39-62)$ & $<0.01$ \\
\hline $\mathrm{Ct}(\mathrm{IQR})$ & $\begin{array}{c}19.58 \\
(17.19-22.49)\end{array}$ & $\begin{array}{c}18.81 \\
(15.72-21.24)\end{array}$ & $\begin{array}{c}19.00 \\
(16.70-20.83)\end{array}$ & 0.72 \\
\hline \multicolumn{5}{|l|}{ Vaccine administered } \\
\hline Astra Zeneca/Oxford & $5(21.73)$ & $3(10.00)$ & $0(0.00)$ & 0.50 \\
\hline Pfizer/BioNTech & $5(21.73)$ & $3(10.00)$ & $0(0.00)$ & 0.50 \\
\hline CanSino & $4(17.39)$ & $20(66.66)$ & $0(0.00)$ & $<0.01$ \\
\hline Sinovac & $6(26.08)$ & $4(13.33)$ & $0(0.00)$ & 0.50 \\
\hline Unspecified & $3(13.04)$ & $0(0.00)$ & $0(0.00)$ & \\
\hline $\begin{array}{l}\text { Complete vaccination } \\
\text { scheme }\end{array}$ & $17(73.91)$ & $29(96.66)$ & $0(0.00)$ & 0.03 \\
\hline $\begin{array}{c}\text { Supplementary oxygen } \\
\text { at arrival time }\end{array}$ & $14(60.86)$ & $0(0.00)$ & $11(57.89)$ & \\
\hline High-flow $\mathrm{O}_{2}$ & $14(60.86)$ & $0(0.00)$ & $6(31.57)$ & 0.07 \\
\hline Intubation & $10(43.47)$ & $0(0.00)$ & $8(42.10)$ & \\
\hline ICU admission & $1(4.34)$ & $0(0.00)$ & $0(0.00)$ & \\
\hline \multicolumn{5}{|l|}{ Comorbidities } \\
\hline Hypertension & $11(47.82)$ & $5(16.66)$ & $3(15.78)$ & 0.09 \\
\hline Diabetes mellitus type II & $13(56.52)$ & $5(16.66)$ & $5(26.31)$ & $<0.01$ \\
\hline Obesity & $1(4.34)$ & $2(6.66)$ & $3(15.78)$ & 0.37 \\
\hline Smoking & $4(17.39)$ & $1(3.33)$ & $2(10.52)$ & 0.23 \\
\hline \multicolumn{5}{|l|}{ Treatment } \\
\hline Tocilizumab & $1(4.34)$ & $0(0.00)$ & $0(0.00)$ & \\
\hline Baricitinib & $16(69.56)$ & $0(0.00)$ & $9(47.36)$ & 0.21 \\
\hline Dexamethasone & $19(82.60)$ & $0(0.00)$ & $12(63.15)$ & 0.27 \\
\hline Methylprednisolone & $17(73.91)$ & $0(0.00)$ & $12(63.15)$ & 0.52 \\
\hline Mortality & $4(17.39)$ & $0(0.00)$ & $3(15.78)$ & \\
\hline
\end{tabular}

${ }^{1}$ IQR: interquartile range; ICU: intensive care unit. ${ }^{2}$ Significant $p$-values are denoted in bold letters.

\subsection{SARS-CoV-2 Lineages and Variants}

At the time of the study, the VOCs were Alpha (B.1.1.7), Beta (B.1.351, B.1.351.2, and B.1.351.3), Gamma (P.1, P.1.1, and P.1.2), and Delta (B.1.617.2, AY.1, AY.2, AY.3, and AY.4). The VOIs were Eta (B.1.525), Iota (B.1.526), Kappa (B.1.617.1), Lambda (C.37), and Mu (B.1.621) [11]. Thus, the comparison studies were performed using this classification.

SARS-CoV-2 variants detected in vaccine breakthrough infections were Delta $(n=36$, $67.92 \%)$, Gamma $(n=4,7.55 \%)$, Mu $(n=4,7.55 \%)$, and Alpha $(n=3,5.66 \%)$, which spanned through the phylogenetic tree shown in Figure 1A. In contrast, Delta $(n=17$, $89.5 \%)$ and Gamma $(n=2,10.5 \%)$ were detected in non-vaccinated hospitalized patients. In breakthrough infections, the four Gamma viruses detected infected patients regardless of their status (ambulatory or hospitalized, vaccinated or non-vaccinated) in our population, and were related to independent introductions (Figure 1B). Mexican Gamma isolates 
spanned throughout the Gamma clade. Viruses isolated in this study did not group directly with other Mexican isolates. In our study, only three Alpha viruses were detected: two in ambulatory patients and the other in a vaccinated hospitalized patient. However, only one Alpha virus could be analyzed due to sequence quality (Figure 1C). Mexican Alpha isolates distributed similarly to Gamma isolates, thus confirming co-circulation of these VOCs. Four Mu viruses were detected: two in vaccinated hospitalized patients and two in ambulatory patients. None were detected in non-vaccinated patients. Three of the $\mathrm{Mu}$ viruses were clustered in the same clade (Figure 1D). At the time of analysis, there were only two more Mexican Mu isolates. One isolate grouped with one of the isolated reported here, suggesting a transmission event. The other isolate was unrelated.

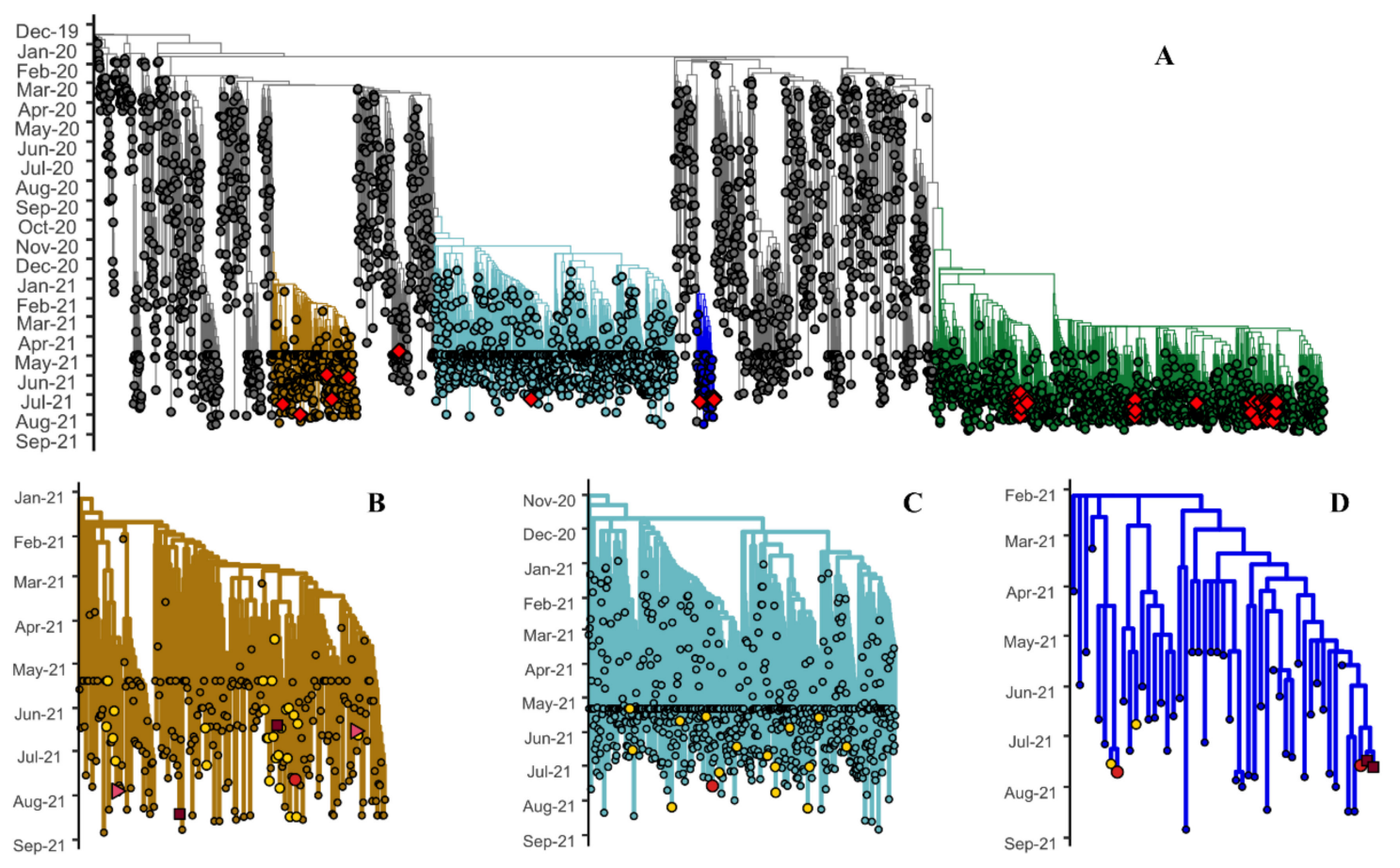

Figure 1. Phylogenetic relationship of SARS-CoV-2, 2019-2021. Global context tree highlighting the different variants: (A) Gamma (gold), Alpha (teal), Mu (blue), and Delta (green). Red diamonds indicate the viruses obtained in this study. The relationships of the different variants detected in the studied patients were magnified into (B) Gamma, (C) Alpha, and (D) Mu. Yellow circles indicate viruses isolated in Mexico. Red circles indicate ambulatory vaccinated patients. Red squares indicate hospitalized vaccinated patients. Pink triangles indicate hospitalized non-vaccinated patients. Sequences used in this analysis were downloaded from the Global Initiative for Sharing All Influenza Data (GISAID) database.

Different Delta strains were detected and distributed throughout the tree (Figure 2A). Delta sequences from Mexico grouped into specific clades, which suggested few introductions. Our sequenced viruses grouped into five clades (Figure 2). The first clade contained 20 samples from ambulatory, hospitalized, and non-vaccinated hospitalized patients (Figure 2B). Within this clade, 13 of the 20 isolated viruses were clustered into a single subclade, indicating a transmission chain. The second clade contained 11 samples from our study (Figure 2F), and the remaining three clades consisted of several viruses (Figure 2C-E). Delta isolated viruses were detected in several Mexican states, thus confirming the wide spread of this variant in Mexico. The frequency of VOCs was $81.13 \%$ $(n=43)$ in all vaccinated patients included in the study. An upward trend was observed in vaccinated patients who were infected by the Delta variant $(p=0.08)$, as well as in those who contracted an infection by VOCs $(p=0.05)$, compared to patients who were not vaccinated. 


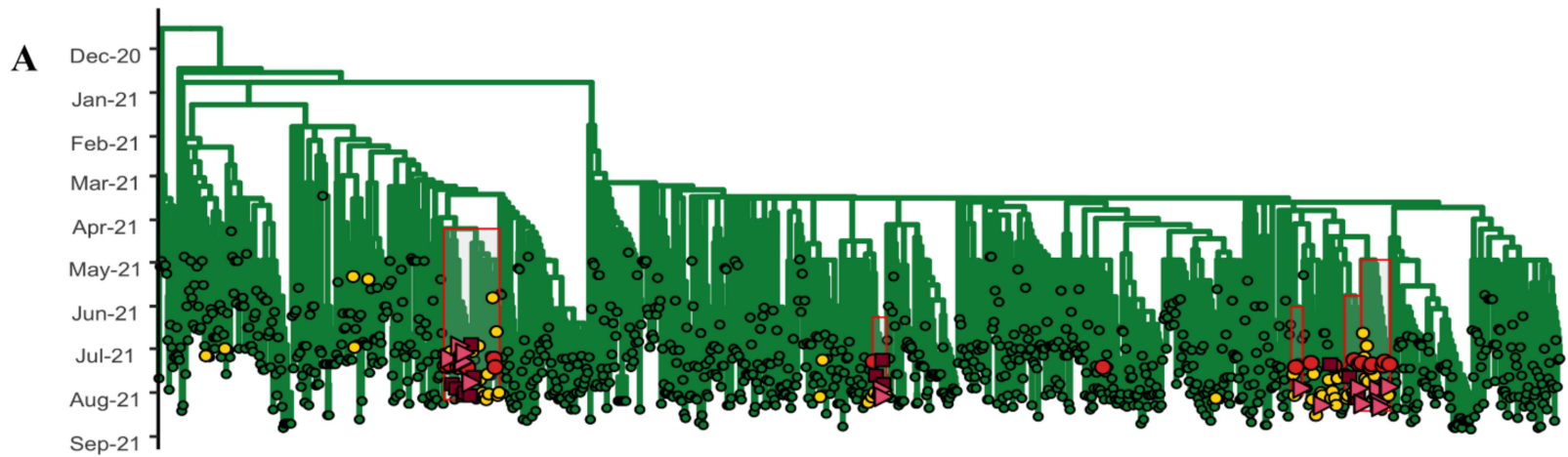

B

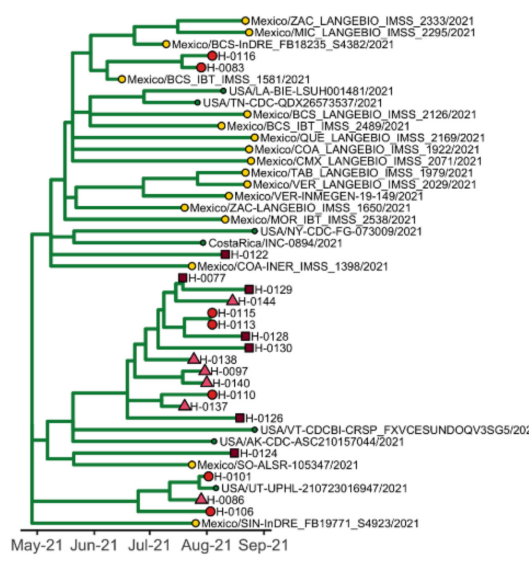

C

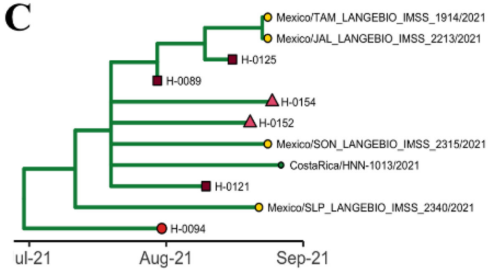

D

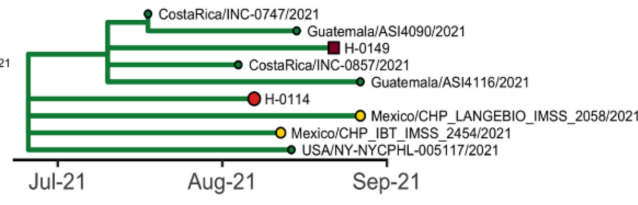

$\mathbf{E}$
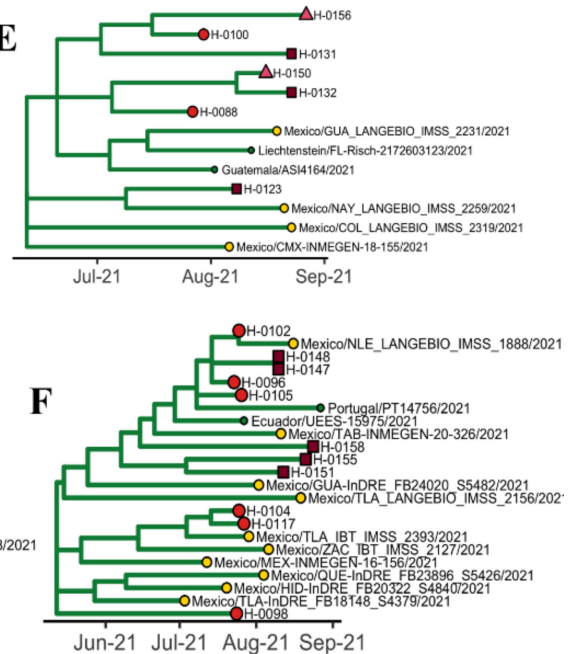

Figure 2. Delta variant phylogenetic relationship. Phylogenetic tree showing the analyzed Delta viruses and the distribution of the obtained sequences (A). Red boxes indicate posterior magnification of the analyzed clades, which are the following: the largest clade involving 20 of the generated sequences (B) and the minor clades in which the obtained sequences grouped with other Mexican isolates (C-F). Yellow circles indicate viruses isolated in Mexico. Red circles indicate ambulatory vaccinated patients. Red squares indicate hospitalized vaccinated patients. Pink triangles indicate hospitalized non-vaccinated patients. Sequences used in this analysis were downloaded from the Global Initiative for Sharing All Influenza Data (GISAID) database.

Detected SARS-CoV-2 lineages were highly diverse (Table 2). B.1.617.2 (Delta variant) predominated in vaccinated ambulatory patients $(n=15,68.18 \%, p<0.01)$ compared to hospitalized patients regardless of vaccine status. Instead, AY.4 (Delta variant) predominated in either vaccinated or non-vaccinated hospitalized patients compared to ambulatory patients $(p=0.04)$. The distribution of remaining lineages between vaccinated and unvaccinated patients showed no statistical difference, suggesting both groups were similarly affected by circulating SARS-CoV-2 variants. 
Table 2. Descriptive data of SARS-CoV-2 variants and lineages detected in this study.

\begin{tabular}{|c|c|c|c|c|}
\hline \multirow{2}{*}{ Variant or Lineage ${ }^{1}$} & \multicolumn{2}{|c|}{$\begin{array}{c}\text { Vaccinated } \\
n(\% \text { or Range })\end{array}$} & \multirow{2}{*}{$\begin{array}{c}\text { Unvaccinated } \\
n \text { (\% or Range) } \\
\begin{array}{c}\text { Hospitalized } \\
(n=19)\end{array}\end{array}$} & \multirow{2}{*}{$p$-Value ${ }^{2}$} \\
\hline & $\begin{array}{l}\text { Hospitalized } \\
\quad(n=23)\end{array}$ & $\begin{array}{c}\text { Ambulatory } \\
(n=30)\end{array}$ & & \\
\hline Alpha & $1(4.34)$ & $2(6.66)$ & $0(0.00)$ & 0.52 \\
\hline B.1.1.7 & $1(100)$ & $2(100)$ & $0(0.00)$ & \\
\hline Gamma & $2(8.68)$ & $2(6.66)$ & $2(10.52)$ & 0.89 \\
\hline P.1 & $2(100)$ & $1(50.00)$ & $2(100)$ & \\
\hline P.1.1 & $0(0.00)$ & $1(50.00)$ & $0(0.00)$ & \\
\hline Delta & $14(60.86)$ & $22(73.33)$ & 17 (89.47) & 0.11 \\
\hline AY.11 & $5(35.71)$ & $0(0.00)$ & $4(23.52)$ & 0.09 \\
\hline AY.3 & $3(21.42)$ & $6(27.27)$ & $2(11.76)$ & 0.49 \\
\hline AY.4 & $4(28.57)$ & $1(4.54)$ & $6(35.29)$ & 0.04 \\
\hline AY.5 & $0(0.00)$ & $0(0.00)$ & $2(11.76)$ & \\
\hline B.1.617.2 & $2(14.28)$ & 15 (68.18) & $3(17.64)$ & $<0.01$ \\
\hline $\mathrm{Mu}$ & $2(8.68)$ & $2(6.66)$ & $0(0.00)$ & 0.96 \\
\hline B.1.621 & $2(100)$ & $2(100)$ & $0(0.00)$ & \\
\hline Other lineages & 4 (17.39) & $2(6.66)$ & $0(0.00)$ & 0.47 \\
\hline B.1 & $1(25.00)$ & $1(50.00)$ & $0(0.00)$ & \\
\hline B.1.1.519 & $1(25.00)$ & $0(0.00)$ & $0(0.00)$ & \\
\hline B.1.618 & $0(0.00)$ & $1(50.00)$ & $0(0.00)$ & \\
\hline B.1.628 & $1(25.00)$ & $0(0.00)$ & $0(0.00)$ & \\
\hline B.1.632 & $1(25.00)$ & $0(0.00)$ & $0(0.00)$ & \\
\hline VOCs & 17 (73.91) & $26(86.66)$ & 19 (100) & 0.05 \\
\hline VOIs & $2(8.68)$ & $2(6.66)$ & $0(0.00)$ & 0.44 \\
\hline
\end{tabular}

${ }^{1}$ VOCs: variants of concern; VOIs: variants of interest. ${ }^{2}$ Significant $p$ values are denoted in bold letters.

\section{Discussion}

COVID-19 vaccination is one of the most effective methods to control the pandemic [16]. However, vaccinated individuals can still become infected and develop COVID-19 symptoms. In these breakthrough infections, SARS-CoV-2 RNA or antigen is detected $\geq 14$ days after receiving recommended doses of a COVID-19 vaccine [17]. Severe COVID-19 disease can occur in $10 \%$ of individuals with breakthrough infections, in which older age is considered a risk factor [18]. Consequently, lower risk of severe disease is associated with vaccination. Some of the vaccines authorized for emergency use in Mexico (i.e., Pfizer/BioNTech and AstraZeneca/Oxford) offer variable protection levels against SARS-CoV-2 VOCs. After complete immunization, the effectiveness against symptomatic infection of Pfizer/BioNTech decreased from 93.7\% against the Alpha variant to $88.0 \%$ with the Delta variant. Additionally, the effectiveness of AstraZeneca/Oxford declined from $74.5 \%$ against the Alpha variant to $67.0 \%$ with the Delta variant. Nevertheless, after only one dose, both vaccines were only $48.7 \%$ and $30.7 \%$ effective against the Alpha and Delta variants, respectively [19].

After mRNA vaccine administration such as Pfizer or Moderna (mRNA-1273, Moderna, Cambridge, MA, USA), breakthrough confirmed cases were reported worldwide, mainly caused by VOCs such as Alpha B.1.1.7 [20-23] and Beta B.1.351 [21,24], in Israel [20,21], Greece [22], Germany [24], and Belgium [23]. The P1 (Gamma VOC) variant was reported in patients with CoronaVac from Brazil [25]. In the USA, a predominance of B.1.526 (Iota VOI) or B.1.1.7 (Alpha VOC) occurred in breakthrough infections in New York [26,27], Massachusetts [28], and other parts of the country [29]. Delta variants such as B.1.617.2 and AY.3 were reported in Massachusetts [30], in addition to P.1 (Gamma), and B.1.351 (Beta) in Washington state [30]. In our study, widespread transmission of the Gamma variant was detected in Mexico, as up to $80 \%$ of the Gamma viruses were isolated in Mexico. One of the three Alpha viruses was clustered with viruses isolated in Austria and New Zealand.

The $\mathrm{Mu}$ (B.1.621) variant carries several known spike mutations, such as E484K, N501Y, and P681H; and some new mutations, such as R346K, Y144T, Y145S, and 146N. 
This variant was first detected in Colombia in January 2021, where its transmission rapidly increased [31], and it was later imported into Italy from a traveler coming from Colombia in April 2021 [32]. The Mu variant was recently designated as a VOI by the WHO on 30 August 2021. To date, the B.1.621 lineage is present in Colombia, the United States, Spain, the Netherlands, Denmark, Germany, Curacao, and Mexico. Particularly in Mexico, we detected four $\mathrm{Mu}$ viruses, three of which were grouped in the same clade, possibly indicating a single transmission event. The fourth $\mathrm{Mu}$ virus was grouped into another clade with a virus isolated in Puebla, Mexico. These results showed that the Mu variant was introduced into Mexico on different occasions. Mu viruses were also grouped with viruses isolated in Ecuador and Colombia.

The B.1.617.2 Delta variant was first detected in India, later becoming dominant in England [33]. This VOC contains mutations in the $S$ viral protein, which is associated with increased viral transmission and infectivity, in addition to higher hospitalization risk [7]. Delta variants (B.1.617.2 and AY.4) were the cause of most of the breakthrough infections in the Monterrey metropolitan area (67.92\%). Delta viruses were clustered into five clades, most of them with high frequency of viruses previously isolated from different Mexican states. The first clade contained 20 samples in addition to a high number of viruses isolated in Mexico (84\%). Within this clade, 13 of the 20 isolated viruses were clustered into a single subclade, indicating a transmission chain. The second clade also contained a high number Mexican isolates (91\%) and 11 samples from our study, which clustered with viruses isolated from different Mexican states. The remaining Delta viruses' clades were also clustered with viruses isolated in Costa Rica, and Guatemala.

In Mexico, a remarkable decline in COVID-19 cases occurred in early spring 2021, due in part to governmental vaccination campaigns in populations at high risk (i.e., health personnel and elderly people) in late December 2020 and early February 2021 [34]. However, during May and June 2021, COVID-19 cases, hospitalizations, and deaths started to increase nationwide soon after the Delta variant was designated as a VOC by the WHO. The highest peak ever reported in Mexico since the pandemic officially started in the country (first government-confirmed COVID-19 case on 28 February 2020) occurred recently on 12 August 2021, with 24,975 new daily cases and 608 deaths [35]. Likewise, COVID-19 cases in EUA increased 300\% during June and July 2021 caused by the Delta variant, which now has a prevalence of $83.4 \%$ [16,36].

The national response to the COVID-19 health emergency varied considerably within the country in the first months of the pandemic. Mexican states had widely different responses in terms of the implementation and timing of measure policies to mitigate the spread of COVID-19. However, the performance of the state of Nuevo Leon was one of the highest in the country [37], as the state government established policies to promote social distancing, such as the suspension of on-site classes and mass events, the reinforcement of economic restrictions, and the stay-at-home request, before federal measures were implemented. Since then, health policies have been highly dynamic with the subsequent lifting of social distancing measures and public space capacity limits that have contributed to a sustained virus transmission in the region [38].

Vaccination efforts should also be accelerated to reduce community transmission. As of 31 August 2021, the proportion of the total Mexican population fully vaccinated was $26.8 \%$, and $38.4 \%$ in the population over 18 years of age [9]. The risk of infection, severe illness, and death in non-vaccinated people is highly significant [16] and vaccination refusal has also complicated the situation [17].

In the first stages of vaccine administration in Mexico, the administration of Pfizer/ BioNTech vaccines was directed toward prioritized population groups (i.e., health personnel attending COVID-19 patients and elderly people), whereas the teaching staff was later vaccinated with CanSino with the hopeful aim of opening schools soon [34]. However, current trends indicate the level of SARS-CoV-2 community transmission remains high [39]. The increase in case detection rates implies that prevention strategies need to be strength- 
ened [16]. COVID-19 incidence should be monitored in populations of high risk of exposure, such as students and teaching personnel, in addition to healthcare workers $[16,40]$.

Our results showed a higher prevalence of CanSino vaccine administration in our population $(45.28 \%$ ) compared to other vaccine brands in the same period. Between April and May 2021, the national government implemented the administration of the CanSino vaccine exclusively in teaching staff, including the state of Nuevo Leon [41]. By the end of May, $87 \%$ of the teaching personnel in Mexico was fully vaccinated [42]. In addition, ours is a university teaching hospital, in which a great part of the teaching staff is attended.

The majority of patients vaccinated with CanSino who had breakthrough infections (66.66\%) developed milder symptoms of COVID-19 that did not require hospitalization $(p<0.01)$. Although our results were not conclusive regarding other types of vaccines, Pfizer/BioNTech and AstraZeneca/Oxford were $88.0 \%$ and $67.0 \%$ effective in reducing symptomatic Delta infection, respectively [19]. In our study, patients who had a complete vaccination scheme were less likely to develop severe COVID-19 disease requiring hospitalization compared to those who received partial or incomplete immunization.

One limitation of this study is the inclusion of patients with partial vaccination who only had one of the two doses recommended for Pfizer/BioNTech, AstraZeneca/Oxford, or CoronaVac $[6,16]$. However, follow-up of instructions to complete vaccination schemes in people vaccinated with one dose is sometimes complicated. Another limitation is the small sample of patients included in the study. Better analyses could be performed if a higher number of samples can be studied.

In conclusion, SARS-CoV-2 breakthrough infections that occurred in our hospital were mostly in patients vaccinated with CanSino due to a higher prevalence of CanSino vaccine administration in our population compared to other vaccine brands in the same period. In our population, most of the patients vaccinated with CanSino who had breakthrough infections developed mild symptoms, and few patients required hospitalization. Patients who had a complete vaccination scheme were less likely to develop severe COVID-19 disease requiring hospitalization compared to those who received partial or incomplete immunization. Delta variants, mainly B.1.617.2 and AY.4, were the cause of most of the breakthrough infections. The recently designated Mu variant was detected in four of our vaccinated patients. The relatively modest number of sequenced samples poses another limitation for the extrapolation of data to other regions, although a strength of this group lies in the valuable data regarding infections in persons vaccinated with CanSino and Sinovac, for whom data are scant.

Supplementary Materials: The following supporting information can be downloaded at: https: / /www.mdpi.com/article/10.3390/v14010154/s1, Table S1: The authors of sequences used, Table S2: Clinical characteristics of breakthrough infection cases from this study.

Author Contributions: Conceptualization, A.C.-O. and E.P.-A.; methodology, K.A.G.-H.; software, K.A.G.-H.; validation, K.A.G.-H.; formal analysis, K.A.G.-H., D.S.-T. and S.F.-T.; investigation, S.A.L.-S., D.A.-S. and A.M.R.-E.; resources, A.C.-O. and K.A.G.-H.; data curation, K.A.G.-H.; writingoriginal draft preparation, S.F.-T.; writing —review and editing, S.F.-T., K.A.G.-H. and P.B.-I.; visualization, A.C.-O., P.B.-I. and S.F.-T.; supervision, A.C.-O., P.B.-I., D.S.-T. and S.F.-T.; project administration, P.B.-I. All authors have read and agreed to the published version of the manuscript.

Funding: This work was funded by Consejo Nacional de Ciencia y Tecnología (CONACyT) with the following grant number 312328, awarded to Kame A. Galán-Huerta.

Institutional Review Board Statement: The study was conducted according to the guidelines of the Declaration of Helsinki and approved by the Ethics Committee of the University Hospital Dr. José Eleuterio González (protocol code BI20-0004, approved on 26 November 2020).

Informed Consent Statement: Informed consent was obtained from all subjects involved in the study. Written informed consent was obtained from the patient(s) to publish this paper.

Data Availability Statement: The sequences generated were deposited in GISAID(EPI_ISL_4889733_ EPI_ISL_4889791 and EPI_ISL_4926951-EPI_ISL_4926955). 


\begin{abstract}
Acknowledgments: We gratefully acknowledge the authors from the originating laboratories responsible for obtaining the specimens, as well as the submitting laboratories where the genome data were generated and shared via GISAID, on which this research was based (see Supplementary Table S1).
\end{abstract}

Conflicts of Interest: The authors declare no conflict of interest.

\title{
References
}

1. World Health Organization. Coronavirus Disease (COVID-19) Pandemic. Available online: https://www.who.int/directorgeneral/speeches/detail/who-director-general-s-opening-remarks-at-the-media-briefing-on-covid-19---11-march-2020 (accessed on 11 August 2021).

2. Dong, E.; Du, H.; Gardner, L. An interactive web-based dashboard to track COVID-19 in real time. Lancet Infect. Dis. 2020, 20, 533-534. [CrossRef]

3. Dhama, K.; Sharun, K.; Tiwari, R.; Dadar, M.; Malik, Y.S.; Singh, K.P.; Chaicumpa, W. COVID-19, an emerging coronavirus infection: Advances and prospects in designing and developing vaccines, immunotherapeutics, and therapeutics. Hum. Vaccines Immunother. 2020, 16, 1232-1238. [CrossRef] [PubMed]

4. Kaur, S.P.; Gupta, V. COVID-19 Vaccine: A comprehensive status report. Virus Res. 2020, 288, 198114. [CrossRef] [PubMed]

5. Francis, A.I.; Ghany, S.; Gilkes, T.; Umakanthan, S. Review of COVID-19 vaccine subtypes, efficacy and geographical distributions. Postgrad. Med. J. 2021, 1-6. [CrossRef]

6. Garcia-Montero, C.; Fraile-Martinez, O.; Bravo, C.; Torres-Carranza, D.; Sanchez-Trujillo, L.; Gomez-Lahoz, A.M.; Guijarro, L.G.; Garcia-Honduvilla, N.; Asunsolo, A.; Bujan, J.; et al. An updated review of SARS-CoV-2 vaccines and the importance of effective vaccination programs in pandemic times. Vaccines 2021, 9, 433. [CrossRef]

7. Raman, R.; Patel, K.J.; Ranjan, K. COVID-19: Unmasking emerging SARS-CoV-2 variants, vaccines and therapeutic strategies. Biomolecules 2021, 11, 933. [CrossRef]

8. Zhang, Y.; Zeng, G.; Pan, H.; Li, C.; Hu, Y.; Chu, K.; Han, W.; Chen, Z.; Tang, R.; Yin, W.; et al. Safety, tolerability, and immunogenicity of an inactivated SARS-CoV-2 vaccine in healthy adults aged 18-59 years: A randomised, double-blind, placebocontrolled, phase $1 / 2$ clinical trial. Lancet Infect. Dis. 2021, 21, 181-192. [CrossRef]

9. Secretary of Health. 65\% of the Adult Population in Mexico Has at Least One Dose Vaccine against COVID-19. Available online: https://www.gob.mx/salud/prensa/65-de-poblacion-adulta-en-mexico-con-al-menos-una-dosis-contra-covid-19?idiom= es (accessed on 29 September 2021).

10. Krause, P.R.; Fleming, T.R.; Longini, I.M.; Peto, R.; Briand, S.; Heymann, D.L.; Beral, V.; Snape, M.D.; Rees, H.; Ropero, A.M.; et al. SARS-CoV-2 variants and vaccines. N. Engl. J. Med. 2021, 385, 179-186. [CrossRef]

11. World Health Organization Tracking SARS-CoV-2 Variants. Available online: https://www.who.int/en/activities/trackingSARS-CoV-2-variants / (accessed on 11 September 2021).

12. Quick, J. nCoV-2019 Sequencing Protocol v3 (LoCost). Available online: https://protocols.io/view/ncov-2019-sequencingprotocol-v3-locost-bh42j8ye (accessed on 2 September 2021).

13. Rambaut, A.; Holmes, E.C.; O’Toole, A.; Hill, V.; McCrone, J.T.; Ruis, C.; Du Plessis, L.; Pybus, O.G. A dynamic nomenclature proposal for SARS-CoV-2 lineages to assist genomic epidemiology. Nat. Microbiol. 2020, 5, 1403-1407. [CrossRef]

14. Hadfield, J.; Megill, C.; Bell, S.M.; Huddleston, J.; Potter, B.; Callender, C.; Sagulenko, P.; Bedford, T.; Neher, R.A. Nextstrain: Real-time tracking of pathogen evolution. Bioinformatics 2018, 34, 4121-4123. [CrossRef]

15. Yu, G.; Smith, D.K.; Zhu, H.; Guan, Y.; Lam, T.T.-Y. Ggtree: An r package for visualization and annotation of phylogenetic trees with their covariates and other associated data. Methods Ecol. Evol. 2017, 8, 28-36. [CrossRef]

16. Christie, A.; Brooks, J.T.; Hicks, L.A.; Sauber-Schatz, E.K.; Yoder, J.S.; Honein, M.A.; Team, C.C.-R. Guidance for implementing COVID-19 prevention strategies in the context of varying community transmission levels and vaccination coverage. Morb. Mortal. Wkly. Rep. 2021, 70, 1044-1047. [CrossRef] [PubMed]

17. Vasireddy, D.; Vanaparthy, R.; Mohan, G.; Malayala, S.V.; Atluri, P. Review of COVID-19 Variants and COVID-19 Vaccine Efficacy: What the Clinician Should Know? J. Clin. Med. Res. 2021, 13, 317-325. [CrossRef] [PubMed]

18. Butt, A.A.; Nafady-Hego, H.; Chemaitelly, H.; Abou-Samra, A.B.; Khal, A.A.; Coyle, P.V.; Kanaani, Z.A.; Kaleeckal, A.H.; Latif, A.N.; Masalmani, Y.A.; et al. Outcomes among patients with breakthrough SARS-CoV-2 infection after vaccination: Breakthrough SARS-CoV-2 infection. Int. J. Infect. Dis. 2021, 110, 353-358. [CrossRef] [PubMed]

19. Lopez Bernal, J.; Andrews, N.; Gower, C.; Gallagher, E.; Simmons, R.; Thelwall, S.; Stowe, J.; Tessier, E.; Groves, N.; Dabrera, G.; et al. Effectiveness of covid-19 vaccines against the B.1.617.2 (Delta) variant. N. Engl. J. Med. 2021, 385, 585-594. [CrossRef] [PubMed]

20. Bergwerk, M.; Gonen, T.; Lustig, Y.; Amit, S.; Lipsitch, M.; Cohen, C.; Mandelboim, M.; Gal Levin, E.; Rubin, C.; Indenbaum, V.; et al. Covid-19 breakthrough infections in vaccinated health care workers. N. Engl. J. Med. 2021, 385, 1474-1484. [CrossRef] [PubMed]

21. Kustin, T.; Harel, N.; Finkel, U.; Perchik, S.; Harari, S.; Tahor, M.; Caspi, I.; Levy, R.; Leshchinsky, M.; Ken Dror, S.; et al. Evidence for increased breakthrough rates of SARS-CoV-2 variants of concern in BNT162b2-mRNA-vaccinated individuals. Nat. Med. 2021, 27, 1379-1384. [CrossRef] [PubMed] 
22. Ioannou, P.; Karakonstantis, S.; Astrinaki, E.; Saplamidou, S.; Vitsaxaki, E.; Hamilos, G.; Sourvinos, G.; Kofteridis, D.P. Transmission of SARS-CoV-2 variant B.1.1.7 among vaccinated health care workers. Infect. Dis. 2021, 53, 876-879. [CrossRef] [PubMed]

23. Geysels, D.; Van Damme, P.; Verstrepen, W.; Bruynseels, P.; Janssens, B.; Smits, P.; Naesens, R. SARS-CoV-2 vaccine breakthrough infections among healthcare workers in a large Belgian hospital network. Infect. Control. Hosp. Epidemiol. 2021, 1-2. [CrossRef]

24. Kroidl, I.; Mecklenburg, I.; Schneiderat, P.; Muller, K.; Girl, P.; Wolfel, R.; Sing, A.; Dangel, A.; Wieser, A.; Hoelscher, M. Vaccine breakthrough infection and onward transmission of SARS-CoV-2 Beta (B.1.351) variant, Bavaria, Germany, February to March 2021. Eurosurveillance 2021, 26, 2100673. [CrossRef]

25. Estofolete, C.F.; Banho, C.A.; Campos, G.R.F.; Marques, B.C.; Sacchetto, L.; Ullmann, L.S.; Possebon, F.S.; Machado, L.F.; Syrio, J.D.; Araujo Junior, J.P.; et al. Case study of two post vaccination SARS-CoV-2 infections with P1 variants in CoronaVac vaccinees in Brazil. Viruses 2021, 13, 1237. [CrossRef]

26. Thompson, C.N.; Hughes, S.; Ngai, S.; Baumgartner, J.; Wang, J.C.; McGibbon, E.; Devinney, K.; Luoma, E.; Bertolino, D.; Hwang, C.; et al. Rapid emergence and epidemiologic characteristics of the SARS-CoV-2 B.1.526 variant-New York City, New York, 1 January-5 April 2021. Morb. Mortal. Wkly. Rep. 2021, 70, 712-716. [CrossRef]

27. Duerr, R.; Dimartino, D.; Marier, C.; Zappile, P.; Wang, G.; Lighter, J.; Elbel, B.; Troxel, A.B.; Heguy, A. Dominance of alpha and Iota variants in SARS-CoV-2 vaccine breakthrough infections in New York City. J. Clin. Investig. 2021, 131, e152702. [CrossRef]

28. North, C.M.; Barczak, A.; Goldstein, R.H.; Healy, B.C.; Finkelstein, D.M.; Ding, D.D.; Kim, A.; Boucau, J.; Shaw, B.; Gilbert, R.F.; et al. Determining the incidence of asymptomatic SARS-CoV-2 among Early Recipients of COVID-19 Vaccines: A prospective cohort study of healthcare workers before, during and after Vaccination [DISCOVER-COVID-19]. Clin. Infect. Dis. 2021, 1-4. [CrossRef]

29. Pollett, S.D.; Richard, S.A.; Fries, A.C.; Simons, M.P.; Mende, K.; Lalani, T.; Lee, T.; Chi, S.; Mody, R.; Madar, C.; et al. The SARS-CoV-2 mRNA vaccine breakthrough infection phenotype includes significant symptoms, live virus shedding, and viral genetic diversity. Clin. Infect. Dis. 2021, ciab543. [CrossRef]

30. McEwen, A.E.; Cohen, S.; Bryson-Cahn, C.; Liu, C.; Pergam, S.A.; Lynch, J.; Schippers, A.; Strand, K.; Whimbey, E.; Mani, N.S.; et al. Variants of concern are overrepresented among post-vaccination breakthrough infections of SARS-CoV-2 in Washington State. Clin. Infect. Dis. 2021, ciab581. [CrossRef]

31. Laiton-Donato, K.; Franco-Munoz, C.; Alvarez-Diaz, D.A.; Ruiz-Moreno, H.A.; Usme-Ciro, J.A.; Prada, D.A.; Reales-Gonzalez, J.; Corchuelo, S.; Herrera-Sepulveda, M.T.; Naizaque, J.; et al. Characterization of the emerging B.1.621 variant of interest of SARS-CoV-2. Infect. Genet. Evol. 2021, 95, 105038. [CrossRef]

32. Messali, S.; Bertelli, A.; Campisi, G.; Zani, A.; Ciccozzi, M.; Caruso, A.; Caccuri, F. A cluster of the new SARS-CoV-2 B.1.621 lineage in Italy and sensitivity of the viral isolate to the BNT162b2 vaccine. J. Med. Virol. 2021, 93, 6468-6470. [CrossRef]

33. Mishra, S.; Mindermann, S.; Sharma, M.; Whittaker, C.; Mellan, T.A.; Wilton, T.; Klapsa, D.; Mate, R.; Fritzsche, M.; Zambon, M.; et al. Changing composition of SARS-CoV-2 lineages and rise of Delta variant in England. EClinicalMedicine 2021, $39,101064$. [CrossRef]

34. Government of Mexico. National Policy of Vaccine Administration against SARS-CoV-2 to Prevent COVID-19 in Mexico. Available online: http:/ /vacunacovid.gob.mx/wordpress/wp-content/uploads/2021/09/2021.09.28-PNVx_COVID-1.pdf (accessed on 28 September 2021).

35. Government of Mexico. COVID-19 Daily Technical Report in Mexico. (In Spanish). Available online: https://www.gob.mx/cms/ uploads/attachment/file/661859/Comunicado_Tecnico_Diario_COVID-19_2021.08.12.pdf (accessed on 2 September 2021).

36. Centers for Disease Control and Prevention. CDC COVID Data Tracker. Available online: https:/ / covid.cdc.gov/covid-datatracker/\#variant-proportions (accessed on 10 August 2021).

37. Knaul, F.; Arreola-Ornelas, H.; Porteny, T.; Touchton, M.; Sanchez-Talanquer, M.; Mendez, O.; Chertorivski, S.; Ortega, S.; Chudnovsky, M.; Kuri, P.; et al. Not far enough: Public health policies to combat COVID-19 in Mexico's states. PLoS ONE 2021, 16, e0251722. [CrossRef]

38. Tariq, A.; Banda, J.M.; Skums, P.; Dahal, S.; Castillo-Garsow, C.; Espinoza, B.; Brizuela, N.G.; Saenz, R.A.; Kirpich, A.; Luo, R.; et al. Transmission dynamics and forecasts of the COVID-19 pandemic in Mexico, March-December 2020. PLoS ONE 2021, 16, e0254826. [CrossRef] [PubMed]

39. Government of Mexico. In Daily Technical Report. Available online: https://coronavirus.gob.mx/2021/08/12/informacion-al12-de-agosto/ (accessed on 2 September 2021).

40. Paltiel, A.D.; Zheng, A.; Walensky, R.P. Assessment of SARS-CoV-2 Screening strategies to permit the safe reopening of college campuses in the United States. JAMA Netw. Open 2020, 3, e2016818. [CrossRef] [PubMed]

41. Vaccine Administration Progress in Mexico up to 28 August 2021. Available online: https://datos.nexos.com.mx/como-vael-avance-en-la-aplicacion-de-vacunas-contra-covid-19-en-mexico-corte-al-28-de-agosto-de-2021/ (accessed on 30 December 2021)

42. Vaccination to Teaching and Administrative Staff Starts Tomorrow on Nuevo Leon State, 26 April 2021. Available online: https:/ / www.gob.mx/sep/articulos/boletin-sep-no-84-inicia-manana-jornada-de-vacunacion-para-docentes-personaladministrativo-y-de-apoyo-en-el-estado-de-nuevo-leon (accessed on 30 December 2021). 